\title{
Symptoms of AF and HF improved by cardioversion
}

The management of patients with concurrent atrial fibrillation and heart failure is challenging. Large trials comparing rate control with rhythm control for atrial fibrillation have tended to exclude patients with heart failure; however, data from small, observational studies indicate that patients with both conditions could benefit from the restoration of sinus rhythm. A team from the UK has now demonstrated, in a randomized, controlled trial, that rhythm control improves left ventricular function and quality of life, and reduces levels of $\mathrm{N}$-terminal prohormone brain natriuretic peptide (NT-proBNP), in patients with atrial fibrillation and heart failure.

The investigators randomly assigned 61 patients with persistent atrial fibrillation, systolic dysfunction, and NYHA class II-IV heart failure to treatment with digoxin and $\beta$-blockers (rate control) or to amiodarone with external electrical cardioversion, as necessary (rhythm control). All patients were anticoagulated with warfarin to an international normalized ratio of 2.0-3.0. The mean age of enrolled patients was 72.4 years and the majority were already taking loop diuretics, $\beta$-blockers, or angiotensin-converting-enzyme inhibitors at baseline.

\section{We need to identify new and effective means of keeping patients with heart failure in sinus rhythm 77}

At the 1-year follow-up, $90 \%$ of patients in the rate-control group had achieved the target heart rate, but all were in atrial fibrillation, and $66 \%$ of those in the rhythm-control group were in sinus rhythm at this time. Rhythm control was associated with significant improvements in left ventricular function and levels of NT-proBNP when compared with rate control, particularly among those individuals who maintained sinus rhythm at 1 year. Quality of life, as assessed with the Medical Outcomes Study Short Form-36 questionnaire, was also significantly better in the rhythm-control group than in the rate-control group. "We need to identify new and effective means of keeping patients with heart failure in sinus rhythm," says Dr Rhidian Shelton, who was one of the investigators. "Our results are from a relatively small cohort of patients ... we await the results of further analysis from the AF-CHF study with respect to quality-of-life data."

\section{Alexandra King}

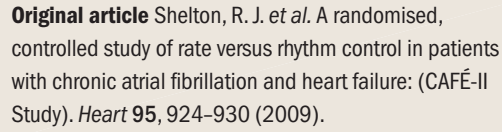

Original article Shelton, R. J. et al. A randomised, controlled study of rate versus rhythm control in patients with chronic atrial fibrillation and heart failure: (CAFÉ-II Study). Heart 95, 924-930 (2009). 